\title{
In situ Mechanical Testing of Contacts Between Nanoscale Bodies: Measuring the Load-dependence of Contact Area.
}

\author{
Sai Bharadwaj Vishnubhotla ${ }^{1}$, Rimei Chen ${ }^{2}$, Subarna R. Khanal ${ }^{1}$, Xiaoli Hu ${ }^{2}$, Ashlie Martini ${ }^{2}$, Tevis D. \\ B. Jacobs ${ }^{1}$ \\ 1. Mechanical Engineering and Materials Science, University of Pittsburgh, Pittsburgh, PA, USA. \\ 2. School of Engineering, University of California-Merced, Merced, CA, USA
}

Contacts between nanoscale bodies can be found in many advanced technologies - including nanomechanical switches and devices [1], catalyst nanoparticles bound to substrates [2], and probe-based manufacturing [3] and microscopy [4]. In these applications, performance and reliability often depend on the nanomechanical response of the nanocontacts: the degree of elastic and inelastic deformation; the strength of adhesive interactions; and the area of contact. However, the mechanical behavior of nanocontacts, and particularly the load-dependence of contact area, is not well understood [5]. These contacts are variously described using (a) continuum mechanics models of spherical bodies; (b) continuum models of rough surfaces with multiple contact points; and (c) fully atomistic descriptions. Experimental evidence for each of these descriptions is largely indirect and inferred from other properties measurements.

Here we perform mechanical testing on a nanoscale contact inside of a transmission electron microscope (TEM), as shown in Fig. 1(a). Using an in situ nanoindenter (Picoindenter PI-95, Hysitron, Inc.), nanoscale bodies composed of silicon and diamond were tested inside of a TEM (2100F, JEOL) with real-time measurements of load, deformation, and contact size [6]. Prior to initiation of testing, the initially-present oxide on the silicon tip was mechanically removed in vacuum, leaving a pure silicon material with a 1$\mathrm{nm}$ amorphous outer layer. Unlike prior in situ TEM investigations of tip-sample contacts, the present tests employed closed-loop displacement control using a capacitive sensor with a feedback rate of $78 \mathrm{kHz}$. The contact was compressed and then pulled until separation at a rate of $1.0 \mathrm{~nm} / \mathrm{s}$. High-resolution TEM images of the nanoscale bodies were captured before and after testing to characterize their materials and geometry. Real-time video was captured during the loading and unloading to measure deformation and contact area using image processing. Further, using the TEM-based materials characterization, an atomistic model tip was created with identically matched materials, geometry, crystallographic orientation, and degree of amorphization. The model tip was loaded and unloaded under similar conditions using molecular dynamics simulations [6], enabling direct comparison and co-validation of results.

The load-dependent contact area was measured directly from the video frames showing the contact. In the simulations, the contact area was computed using several different criteria (described in Ref. [5]), and demonstrated significant variation based on method used. Using a side-view diameter and assuming a circular contact, in direct analogy to the in situ TEM method, the measured contact area agreed within uncertainty between simulations and experiments, as shown in Fig. 1(b). In experiment and simulation, the contact area showed significant hysteresis. During unloading, the contact area at zero applied load was larger by $79 \%$ (in experiments) and 101\% (in simulations) than the same value measured during loading.

The load-dependent contact area during unloading could be accurately fit using continuum contact mechanics models. Specifically, the appropriate contact mechanics model was determined [7] using the measured values of tip radius and pull-off force. The present contact was in the intermediate regime between the simple DMT and JKR limits [8], and was thus described using a numerical implementation [9] of the Maugis-Dugdale model [8]. Using only the unloading data, the experimental and simulation 
data were fit separately, with the effective modulus $E_{\text {eff }}$ as a fit parameter. The best-fit curves are shown in Fig. 1(b) and yielded values of $E_{\text {eff }}=109 \mathrm{GPa}$ and $87 \mathrm{GPa}$ for experiments and simulations, respectively. Both of these values fall between the expected effective moduli using crystalline silicon (127 GPa in the present loading orientation) and amorphous silicon (78 GPa [10]). These best-fit curves cannot be used to fit the loading data; they overestimate the contact area by an average of $40 \%$. Moreover, there was no set of model parameters found that accurately fit the loading portion of the curve using the Maugis-Dugdale, DMT, or JKR models. While plastic deformation can cause hysteretic behavior, it did not appear to be a significant factor here. There was no significant shape change in the tip upon testing (the tip radius was $20.0 \pm 1.8 \mathrm{~nm}$ before and $20.7 \pm 1.4 \mathrm{~nm}$ after), and no sub-surface lattice defects were observed after testing. Further, the experimental tip had been preloaded to an equal force immediately before this test was done.

In conclusion, mechanical tests were performed on a silicon/diamond nanocontact. Using in situ TEM and matched atomistic simulations, the contact area was measured during loading and unloading. The results agreed within uncertainty, and both experiment and simulation data showed significant hysteresis. While the unloading curves could be fit to a continuum model, yielding a realistic value for elastic modulus, this model overpredicted the contact area upon loading by an average of $40 \%$. The implications of these results for real-world nanoscale contacts are that the contact area upon loading can deviate significantly from continuum predictions, even when the behavior upon unloading is well described by these models. [11]

\section{References:}

[1] OY Loh and HD Espinosa, Nat. Nanotechnol. 7 (2012), p. 283.

[2] JA Farmer and CT Campbell, Science 329 (2010), p. 933.

[3] D Pires, et al, Science 328 (2010) p. 732.

[4] E Meyer, HJ Hug and R Bennewitz, "Scanning Probe Microscopy: Lab on a Tip" (Springer, Berlin).

[5] TDB Jacobs and A Martini, Appl. Mech. Rev., Accepted (2017).

[6] SB Vishnubhotla, et al, Tribol. Lett., Under Review (2017).

[7] DS Grierson, EE Flater and RW Carpick, J. Adhes. Sci. Technol. 19 (2005), p. 291.

[8] D Maugis, J. Colloid Interface Sci. 150 (1992), p. 243.

[9] RW Carpick, DF Ogletree and M Salmeron, J. Colloid Interface Sci. 211 (1999) p. 395.

[10] LB Freund and S Suresh, "Thin Film Materials: Stress, Defect Formation and Surface Evolution" (Cambridge University Press, Cambridge).

[11] This work was funded by the National Science Foundation, through CMMI-1536800 and 1537613.
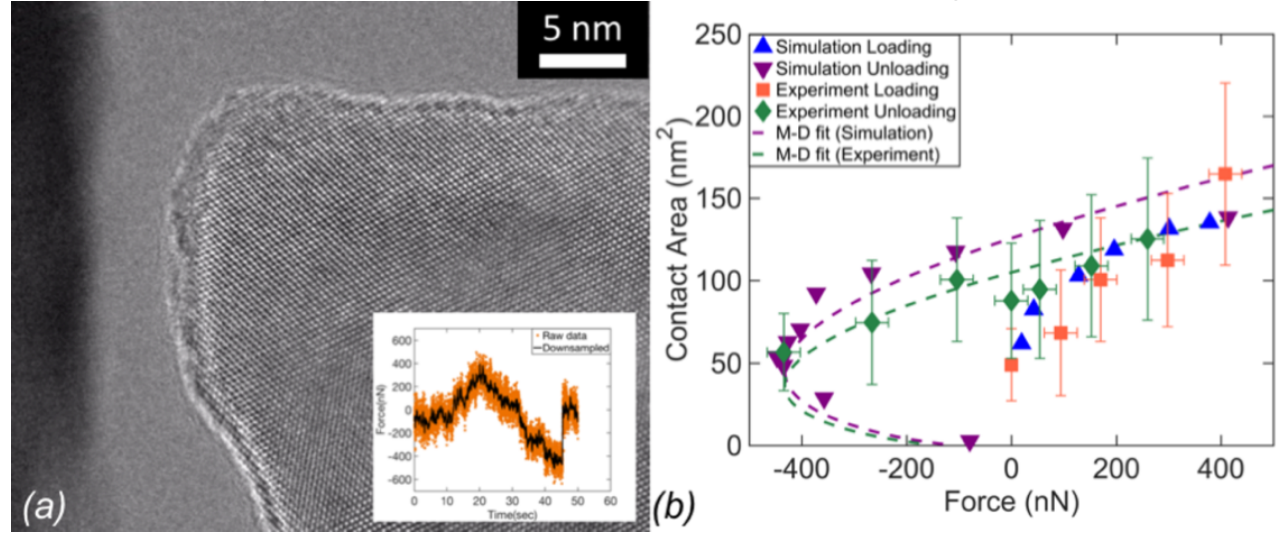

Figure 1. (a) A diamond indenter (left) is brought into contact with a nanoscale silicon asperity (right) while measuring load (inset). (b) The load-dependent contact area is measured in situ and from matched simulations. Results are hysteretic, and can be fit to continuum models during unloading but not loading. 